\title{
Bovine surfactant in the treatment of pneumonia-induced-neonatal acute respiratory distress syndrome (NARDS) in neonates beyond 34 weeks of gestation: a multicentre, randomized, assessor-blinded, placebo-controlled trial
}

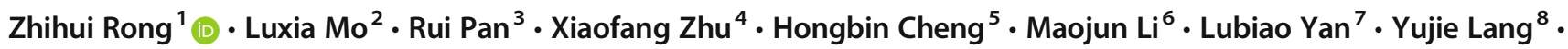 \\ Xiaoshan Zhu ${ }^{9} \cdot$ Liping Chen $^{10} \cdot$ Shiwen Xia ${ }^{11}$. Jun Han ${ }^{12} \cdot$ Liwen Chang ${ }^{1}$
}

Received: 6 May 2020 / Revised: 21 September 2020 / Accepted: 24 September 2020 / Published online: 21 October 2020

(C) Springer-Verlag GmbH Germany, part of Springer Nature 2020

\begin{abstract}
Neonatal acute respiratory distress syndrome (NARDS) reflects pulmonary surfactant dysfunction, and the usage of bovine surfactant (Calsurf) supplement may therefore be beneficial. To determine whether bovine surfactant given in NARDS can improve oxygenation and survival rate, we conducted a multicenter, randomized trial between January 2018 and June 2019, and we compared Calsurf treatment to controls in neonates with pneumonia accompanied by NARDS. Neonates who met the Montreux criteria definition of NARDS were included, and those with congenital heart and lung malformations were excluded. Primary outcomes were oxygenation index (OI) after Calsurf administration, and secondary outcomes were mortality, and duration of ventilator and oxygen between the two groups, and also other morbidities. Cumulatively, 328 neonates were recruited and analyzed, 162 in the control group, and 166 in the Calsurf group. The results shows that OI in the Calsurf group were significantly lower than that in the control group at $4 \mathrm{~h}(7.2 \pm 2.7$ and $11.4 \pm 9.1, P=0.001)$; similarly, OI in the Calsurf group were significantly lower than in the control group at $12 \mathrm{~h}(7.5 \pm 3.1$ and $11.2 \pm 9.2, P=0.001)$. Mortality and duration of ventilator support or oxygen use between the two groups were not significantly different.

Conclusion: Calsurf acutely improved OI immediately after administration in pneumonia-induced NARDS; although, we observed no significant decrease in mortality, duration of ventilator or oxygen, or major morbidity.

What is known:

- The definition proposed as the Monteux criteria for neonatal acute respiratory distress syndrome (NARDS).

- Surfactant acutely improved oxygenation and significantly decreased mortality in children and adolescents with acute lung injury.

What is new:

- This is the first large randomized controlled trail to study on surfactant treatment of neonates with acute respiratory distress syndromes.

- Surfactant acutely improved oxygenation immediately after administration in pneumonia-induced NARDS at a gestational age beyond 34 weeks
\end{abstract}

Keywords Acute respiratory distress syndrome $\cdot$ Neonate $\cdot$ Oxygenation index

Zhihui Rong, Luxia Mo, Rui Pan, Xiaofang Zhu, Hongbin Cheng, Maojun Li, Lubiao Yan, Yujie Lang, Xiaoshan Zhu, Liping Chen, Shiwen Xia and Jun Han have contributed equally to this work and shall be considered co-first authors

Communicated by Daniele De Luca

Electronic supplementary material The online version of this article (https://doi.org/10.1007/s00431-020-03821-2) contains supplementary material, which is available to authorized users.

Liwen Chang

13871530925@163.com

Extended author information available on the last page of the article
Abbreviations

ARDS Acute respiratory distress syndrome

ECMO Extracorporeal membrane oxygenation

HFV High-frequency oscillatory ventilation

IVH Grade 3/4 intracranial ventricular hemorrhage

NARDS Neonatal acute respiratory distress syndrome

NEC Necrotizing enterocolitis

OI Oxygenation index

pARDS Pediatric acute respiratory distress syndrome

PPRPM Prolonged premature rupture of membrane

PVL Periventricular leukomalacia

SGA Small-for-gestational-age 


\section{Introduction}

In 2015, the Pediatric Acute Lung Injury (ALI) Consensus Conference 2015 (PALICC) developed diagnostic criteria for acute respiratory distress syndrome (ARDS) in children ranging from infants to teenagers. ARDS is a generalized inflammatory response of the lung to catastrophic events of various pulmonary and non-pulmonary origins, such that children with ARDS often exhibit high mortality [1]. It is generally accepted that the degree of hypoxemia predicts outcome, mortality in children with a $\mathrm{PaO}_{2} / \mathrm{FiO}_{2}$ ratio lower than 100 greater than $50 \%$. Another outcome predictor is ventilation support, reflected in mean airway pressure [2]. There are many ventilation strategies being evaluated in the pediatric age group to prevent barotrauma and volutrauma, including high-frequency oscillatory ventilation (HFV), inhaled nitric oxide, extracorporeal membrane oxygenation (ECMO), and surfactant treatment [3].

In 2017, De Luca et al. [4] proposed the Monteux definition for neonatal acute respiratory distress syndrome (NARDS). But information regarding NARDS - especially treatment strategies - is still limited. Chinese clinicians performed several single-center clinical studies that were published in Chinese journals [16, 17], finding that Calsurf (Shuanghe Pharmaceutics, Beijing, China) supplement benefited infants with NARDS. However, to our knowledge, there is still paucity of evidence from randomized controlled trials to support the application of surfactant in NARDS. Thus, we performed a randomized, multicenter, assessor-blinded, placebo-controlled trial in patients with NARDS induced by pneumonia to better understand whether oxygenation and survival rate improve after exogenous surfactant administration in cases of mild, moderate, and severe NARDS.

\section{Methods}

\section{Patients}

The present study was a randomized control trial (ChiCTROPC-17011351) conducted at 12 level 3 neonatal intensive care units between January 2018 and June 2019. The distribution of the 12 centers is depicted in Supplementary Data, and the study protocol was approved by the review boards of these institutes. Informed consent was obtained from a parent or guardian before enrollment.

Entry criteria included (1) a gestational age above 34 weeks and (2) an acute onset of pneumonia no later than 1 week of age. The definition of neonates pneumonia was according to Dell'Orto's report: presented respiratory distress with either patchy infiltrates and/or irregular opacities at chest radiogram or loss of aeration/consolidations on lung ultrasound and both of the following criteria: (1) at least one of the following: risk factors for perinatal infection (chorioamnionitis, maternal fever, bacterial colonization, rupture of membranes for more than $18 \mathrm{~h}$ ); (2) at least one of the following: serum $\mathrm{C}$ reactive protein $>10 \mathrm{mg} / \mathrm{L}$, or serum procalcitonin beyond reference values according to hours of life[24]; (3) absence of congenital heart disease that would explain the edema (such as ductus arteriosus with pulmonary overflow if no acute pulmonary hemorrhage existed-echocardiography was needed to verify the origin of the edema); (4) oxygenation deficit expressed as OI, mild NARDS at 4-7.9, moderate NARDS at $8-15.9$, and severe NARDS at $\geq 16$. Exclusion criteria included RDS, TTN, or any congenital anomaly capable of causing a primary, current, acute respiratory condition. These inclusion and exclusion criteria depict a newborn population completely fulfilling the Montreux criteria for the diagnosis of NARDS [4].

\section{Grouping and randomization}

Neonates were assigned randomly to control or Calsurf groups: random numbers were generated by a computer program and randomization performed using serially numbered, opaque, sealed envelopes that were opened by research personnel after obtaining parental consent. According to protocol, a second intervention was performed $12 \mathrm{~h}$ later if the oxygenation index remained higher than 7 .

Although blinding of the clinicians was not possible due to the nature of the intervention, assessors who did data collection and measured OI were blinded to the type of treatment administered.

\section{Methods of surfactant administration}

Calsurf is extracted from lung lavage of calves. Total phosphate lipids are $35 \mathrm{mg} / \mathrm{ml}$, with $59 \%$ of DPPC; $0.78 \mathrm{mg} / \mathrm{m} \mathrm{l}$ of surfactant $B$ and $1.74 \mathrm{mg} / \mathrm{ml}$ of surfactant $C$. The recommended dosage is $100 \mathrm{mg} / \mathrm{kg}$.

For surfactant administration, the position of the endotracheal tube was determined by the length of the tube at the lip, the symmetry of breath sounds, and the symmetric rising of the chest wall. Natural surfactant (Calsurf; Shuanghe Pharmaceutics, China) was refrigerated at $-10{ }^{\circ} \mathrm{C}$ before use and solubilized in $1.5 \mathrm{~mL}$ of sterile water to form a suspension. The suspension was warmed to approximately $37^{\circ} \mathrm{C}$ and then a catheter was used to administer the surfactant near the proximal end of the endotracheal at a dose of $100 \mathrm{mg} / \mathrm{kg}$. The infants were disconnected with the tube and manual bag positive pressure ventilation (maintained for approximately 5 min) was exerted during surfactant administration, followed by mechanical ventilation. The control group received a volume equivalent air bolus in the endotracheal tube, followed by manual bag positive pressure ventilation (maintained for approximately $5 \mathrm{~min}$ ). 


\section{Study outcomes}

Primary outcomes were $\mathrm{OI}$ at 4,12 , and $24 \mathrm{~h}$ after administration of Calsurf. OI was based on pre-ductal arterial $\mathrm{PaO}_{2}$ in all infants included. Secondary outcomes were mortality, duration of ventilator or oxygen, and major morbidity issues, including incidence of pneumothorax, pulmonary hypertension, stage 2 or greater necrotizing enterocolitis (NEC), grade $3 / 4$ intracranial ventricular hemorrhage (IVH), or periventricular leukomalacia (PVL).

We conducted a stratified study, and OI and mortality were compared between pre-specified subgroups.

Small-for-gestational-age (SGA) was defined as birth weight below the 10th percentile at a particular gestational week in Fenton growth chart [5].

\section{Sample size calculation and statistical analysis}

The preliminary data from our center indicated that the OI was 11 in controls, and we hypothesized that treatment would decrease OI to 7 . We therefore required 130 cases and 130 controls to be able to reject the null hypothesis that the exposure rates for cases and controls were equal, with a probability (power) 0.8 . The type I error probability associated with the test of our null hypothesis was 0.05 . We anticipated an attrition of $10 \%$ of infants, and therefore needed to enroll 145 patients were needed in each group.

We used the statistical software packages SPSS for Windows version 19 for data analyses. Continuously distributed variables were expressed as mean \pm one standard deviation, and categorical variables were summarized as numbers and percentages. We used analysis of variance (ANOVA) after analyzing for homogeneity analysis of variance, and this was followed by post hoc testing. The nonparametric rank-sum test (Kruskal-Wallis test) was used if the variance was not homogenous. We performed a multivariate linear regression analysis to rule out confounding factors. Candidate variables with a $p$ value $<0.1$ on univariate analysis and considered clinically relevant were included in multivariable model. Variables for inclusion were carefully chosen, given the number of events available. All the eligible variables were entered into the model. Results are presented as beta and confidence intervals (CI 95\%) and $p$ values. Adjusted $R^{2}$ is presented to evaluate the goodness of fit evaluation. $P<0.05$ was considered to show a significant difference.

\section{Results}

\section{Baseline characteristics and antenatal factors of the two groups}

A total of 345 neonates met the inclusion criteria and provided consent: 172 of these neonates were randomly assigned to the control group and 173 were randomly assigned to the Calsurf treatment group. Ten infants died in the control group and seven infants died in the Calsurf group, and therefore 162 controls and 166 treatments were ultimately analyzed (Fig. 1). Baseline characters and antenatal factors of the two groups were similar, although $P$ values for prenatal steroid usage and gestational age showed a tendency toward significance (Table 1). In the Calsurf treatment group, patients received an average of 3 doses (1 to 8 ) of surfactant.

\section{Primary outcome}

The results between-group patients comparisons shows that $\mathrm{OI}$ in the Calsurf group were significantly lower than in the control group at $4 \mathrm{~h}(7.2 \pm 2.7$ and $11.4 \pm 9.1, P=0.001)$, similarly, OI in the Calsurf group were significantly lower than in the control group at $12 \mathrm{~h}(7.5 \pm 3.1$ and $11.2 \pm 9.2, P$ $=0.001)$ (Fig. 2).

We conducted a stratified study, and the results are shown in Fig. 2. Infants with NARDS were stratified into three categories according to oxygenation deficit expressed as OI: mild NARDS, 4-7.9; moderate NARDS, 8-15.9; and severe NARDS, $\geq 16$. The cases of mild, moderate, and severe NARDS in this study were 105,113 , and 110 , respectively, and Calsurf administration significantly decreased the OI in all 3 groups, especially in moderate and severe cases (Fig. 2). We performed a multivariate linear regression analysis of OI at 4 and $12 \mathrm{~h}$ using subgroup partitioning, and the results are presented in Table 2. When we brought the variables (prenatal steroids, gestational age, and intervention) into the regression model to remove the confounding factors, we observed that only Calsurf treatment was a dependent protective factor in reducing OI.

\section{Secondary outcomes}

The overall mortality rate in NARDS was approximately 5\%, with 10 deaths in control group and 7 deaths in Calsurf group. Duration of ventilation or oxygen and length of hospital stay did not differ between the two groups $(P>0.05)$, and we did not observe a difference in the incidence of pneumothorax, pulmonary hypertension, NEC (stage 2 or greater), grade $3 / 4$ IVH, or PVL (Table 3).

\section{Discussion}

\section{NARDS}

ARDS is defined by radiographic diagnosis of diffuse bilateral alveolar infiltrates, the degree of hypoxemia, lung function, and histopathology, and it occurs in all age groups. 
Table 1 Baseline characteristics and antenatal factors of the two groups

\begin{tabular}{llll}
\hline & Control $(n=162)$ & $\begin{array}{l}\text { Calsurf treatment } \\
(n=166)\end{array}$ & $\begin{array}{l}\text { Univariate } \\
\text { analysis }(P)\end{array}$ \\
\hline Gestational age (weeks), mean (SD) & $37.86(2.6)$ & $37.34(2.1)$ & 0.07 \\
Birth weight (g), mean (SD) & $3090(503)$ & $2985(579)$ & 0.08 \\
Male, $N(\%)$ & $101(62)$ & $108(65)$ & 0.61 \\
Cesarean section, $N(\%)$ & $114(70)$ & $124(75)$ & 0.38 \\
Apgar score at 5 min, mean (SD) & $8(1.6)$ & $8(1.3)$ & 0.12 \\
Antenatal steroid, $N(\%)$ & $13(8)$ & $23(14)$ & 0.09 \\
Pregnancy-induced maternal disease, $N(\%)$ & $40(25)$ & $45(27)$ & 0.91 \\
PPROM, $N(\%)$ & $25(15)$ & $30(18)$ & 0.71 \\
Postnatal hours of NARDS recruitment, mean (SD) & $42.3(50.5)$ & $44.7(52.1)$ & 0.86 \\
OI at study entry & $15.5(11.7)$ & $14.9(11.5)$ & 0.71 \\
Small for gestational age, $N(\%)$ & $5(3.1)$ & $7(4.2)$ & 0.45 \\
\hline
\end{tabular}

PPRPM, prolonged premature rupture of membrane. Values are number (percentage) unless otherwise indicated. $P>0.05$ for all comparisons
ARDS and respiratory distress syndrome (RDS) in neonates show the same clinical characteristics, and differentiation of the two syndromes is justified on the basis of pathophysiologic and etiologic differences [6]. NARDS was not defined in neonates until 2017, when De Luca et al. published an article describing the biologic and pathophysiologic features of NARDS as being similar to those seen in older children and adults with ARDS [4].

Fig. 1 Flow chart of the participants
We only focused on pneumonia-induced ARDS which defines ARDS more accurately especially for late preterm infants. It is generally accepted that the degree of hypoxemia and ventilator support (as reflected by mean airway pressure) can predict outcome [2], and we therefore stratified infants with NARDS into three categories based on OI. This definition stipulates OI thresholds of 4.0-7.9 for mild NARDS, 8.0-15.9 for moderate NARDS, and 16.0 or higher for severe NARDS [7, 8].

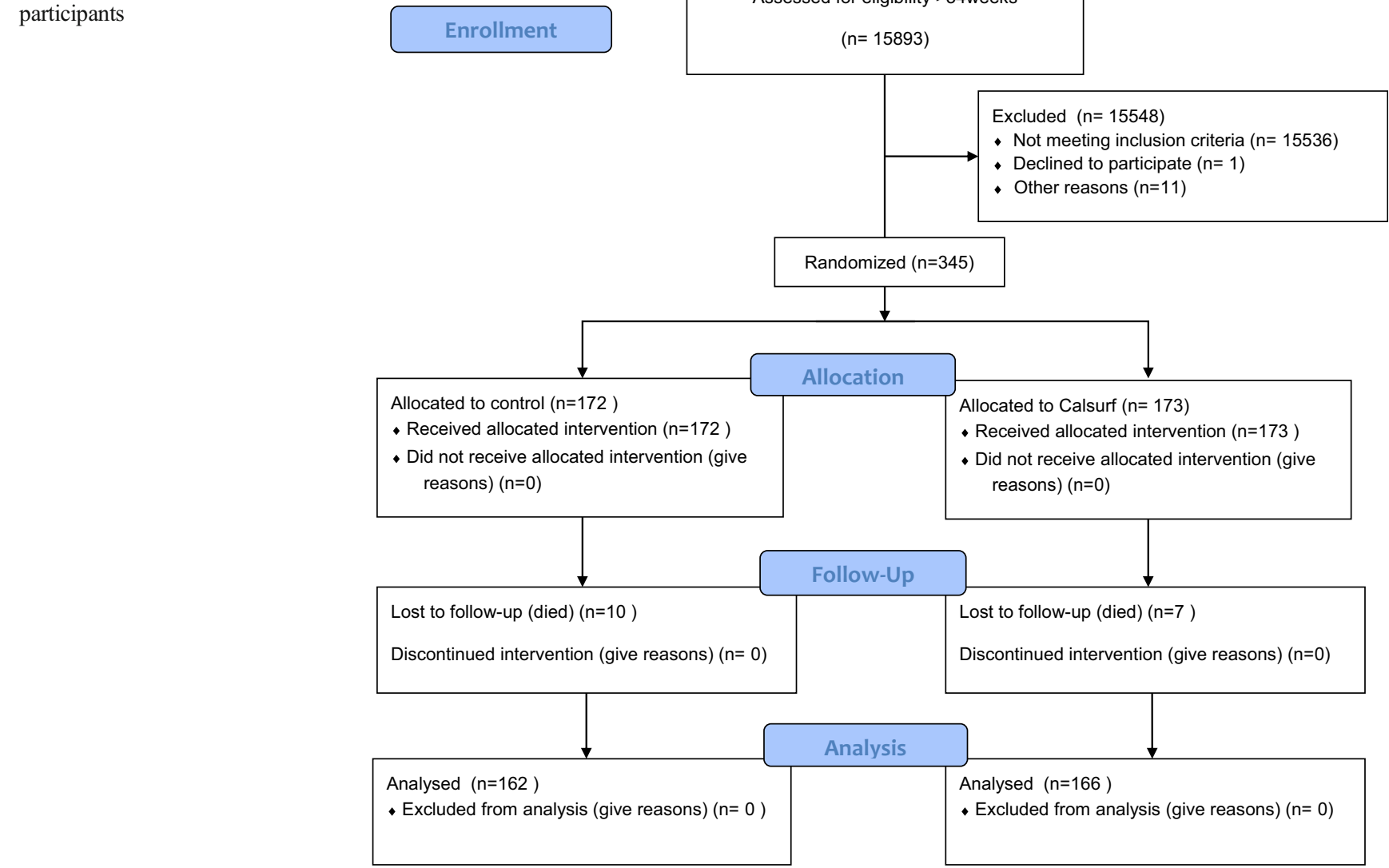



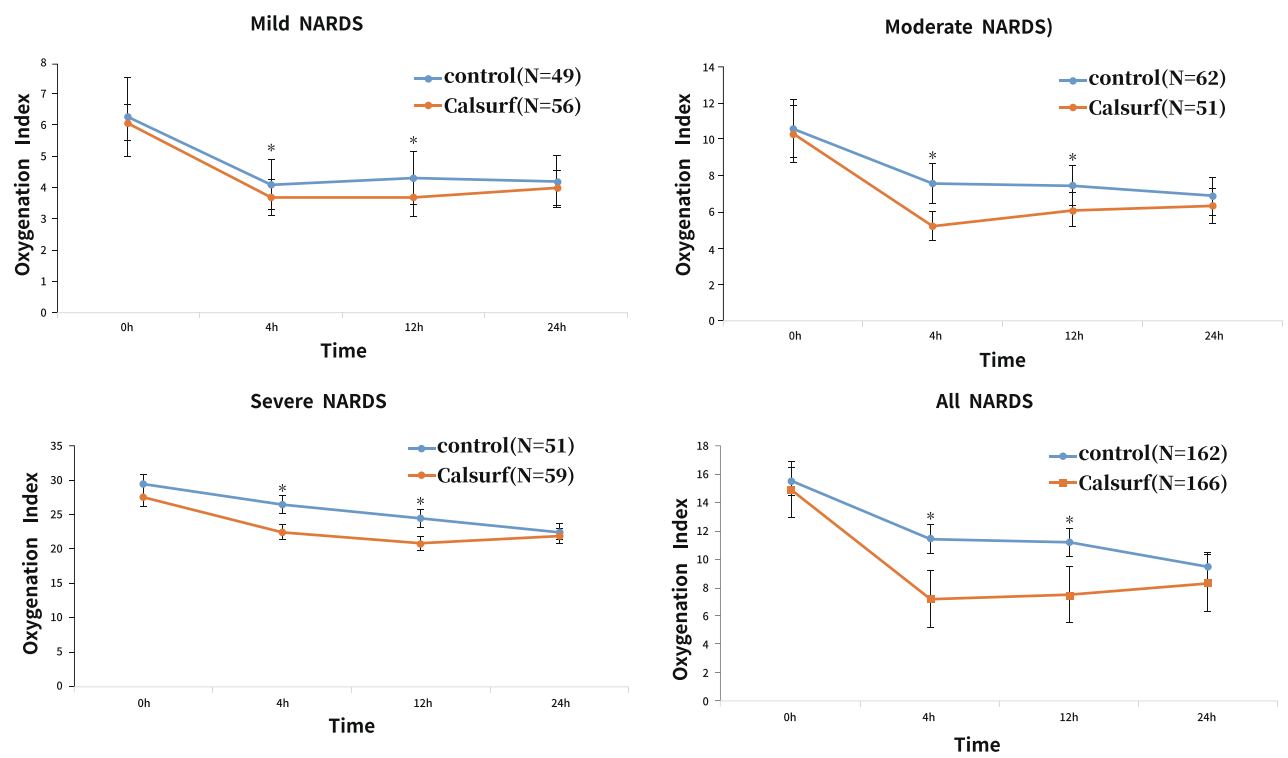

Fig. 2 Oxygen index in mild, moderate and severe NARDS. a mild NARDS; b moderate NARDS; $\mathbf{c}$ severe NARDS; $\mathbf{d}$ total. The overall $p$ value for within patients comparisons: in Calsurf treatment group, OI decreased significantly after $4 \mathrm{~h}$ surfactant given $(7.23 \pm 2.73$ at $4 \mathrm{~h}$, $14.89 \pm 11.44$ at $0 \mathrm{~h}, P<0.001)$ and $12 \mathrm{~h}$ given $(7.53 \pm 3.13$ at $12 \mathrm{~h}$, $14.89 \pm 11.44$ at $0 \mathrm{~h}, P<0.001$ ), while the same change pattern can be

seen in control group, although the decline is not as obvious as control group. The between-group patients' comparisons: Calsurf administration significantly decreased the OI in mild, moderate, and severe cases of NARDS, especially moderate and severe cases. Data shown are mean values; error bars indicate SEM. $* P<0.05$ for the significant difference between groups

\section{Calsurf treatment improves 0 l but has no effect on survival rate}

Many therapies are currently being evaluated in pediatric patients, including lung-protective ventilation strategies, inhaled nitric oxide, extracorporeal membrane oxygenation (ECMO), and surfactant treatment [3]. NARDS is biologically characterized by qualitative or quantitative surfactant dysfunction that affects proteins and phospholipids, and the afflicted neoantes show extensive inflammation of their pulmonary tissues $[9,10]$. Two studies of recombinant surfactant protein in NARDS indicated improvement in oxygenation but without an effect on survival [11,12], and a follow-up analysis of

the study suggested patients with severe NARDS from pneumonia had an improvement in a 28-day survival after surfactant administration [12]. Calsurf is extracted from newborn bovine lungs, is produced in China, and has been used here for several years. Chinese clinical trials by Kong et al. showed that Calsurf supplementation improved pulmonary status when given to preterm infants with severe RDS [13, 14], and Cogo P et al. reported that compared to treatment with $200 \mathrm{mg} / \mathrm{kg}$ of porcine surfactant, $100 \mathrm{mg} / \mathrm{kg}$ of porcine surfactant increased the risk of reuse. However, it is not possible to use such high doses of bovine surfactants; this is one of the major reasons of the inferiority of bovine compared to porcine surfactant [15-17]. Regarding to the selection of drug dosage, Chinese

Table 2 Multivariate linear regression analysis of OI at $4,12 \mathrm{~h}$

\begin{tabular}{|c|c|c|c|c|c|c|c|c|c|c|c|c|}
\hline & \multicolumn{4}{|c|}{ Mild NARDS } & \multicolumn{4}{|c|}{ Moderate NARDS } & \multicolumn{4}{|c|}{ Severe NARDS } \\
\hline & Beta & $95 \% \mathrm{CI}$ & $P$ & Adj. $R^{2}$ & Beta & $95 \% \mathrm{CI}$ & $P$ & Adj. $R^{2}$ & Beta & $95 \% \mathrm{CI}$ & $P$ & Adj. $R^{2}$ \\
\hline $4 \mathrm{~h}$ & & & & 0.06 & & & & 0.37 & & & & 0.65 \\
\hline Gestational age & 0.05 & $-0.02,0.1$ & 0.1 & & -0.1 & $-0.25,0.0$ & 0.0 & & -0.03 & $-0.54,0.48$ & 0.91 & \\
\hline Antenatal steroid & 0.17 & $-0.38,0.71$ & 0.54 & & 0.06 & $-1.45,1.57$ & 0.94 & & 2.02 & $-1.42,5.46$ & 0.25 & \\
\hline Calsurf & -0.46 & $-0.79,-0.12$ & 0.01 & & -2.49 & $-3.11,-1.88$ & 0.00 & & -15.8 & $-18.03,-13.59$ & 0.0 & \\
\hline $12 \mathrm{~h}$ & & & & 0.04 & & & & 0.12 & & & & 0.59 \\
\hline Gestational age & 0.03 & $-0.6,0.13$ & 0.48 & & -0.12 & $-0.27,0.04$ & 0.13 & & 0.00 & $-0.52,0.53$ & 0.99 & \\
\hline Antenatal steroid & 0.22 & $-0.47,0.92$ & 0.52 & & -0.44 & $-2.15,1.26$ & 0.61 & & 1.98 & $-1.54,5.49$ & 0.27 & \\
\hline Calsurf & 0.54 & $0.11-0.97$ & 0.02 & & -1.44 & $-2.13,-0.75$ & 0.0 & & -14.3 & $-16.6,-12.06$ & 0.0 & \\
\hline
\end{tabular}


Table 3 Secondary outcomes in the two groups

\begin{tabular}{|c|c|c|c|c|c|c|c|}
\hline \multirow[t]{2}{*}{ Secondary outcomes } & \multirow[t]{2}{*}{ Control $(n=162)$} & \multirow{2}{*}{$\begin{array}{l}\text { Calsurf treatment } \\
(n=166)\end{array}$} & \multirow{2}{*}{$\begin{array}{l}\text { Univariate } \\
\text { analysis }(P)\end{array}$} & \multicolumn{4}{|c|}{ Multivariate analysis } \\
\hline & & & & Confounders & Beta & $95 \% \mathrm{CI}$ & $P$ \\
\hline \multirow{3}{*}{$\begin{array}{l}\text { Hours on mechanical } \\
\text { ventilator, mean (SD) }\end{array}$} & \multirow[t]{3}{*}{$92.1(42.8)$} & \multirow[t]{3}{*}{$88.8(71.1)$} & \multirow[t]{3}{*}{0.625} & Gestational age & -0.190 & $-2.819,2.438$ & .887 \\
\hline & & & & Antenatal steroid & -28.768 & $-48.911,-8.625$ & .005 \\
\hline & & & & Calsurf & -1.773 & $-13.939,10.392$ & .774 \\
\hline \multirow{3}{*}{$\begin{array}{l}\text { Ventilator free days, } \\
\text { mean (SD) }\end{array}$} & \multirow[t]{3}{*}{$8.8(4.9)$} & \multirow[t]{3}{*}{$10.1(6.7)$} & \multirow[t]{3}{*}{0.163} & Gestational age & 0.008 & $-.102, .117$ & .887 \\
\hline & & & & Antenatal steroid & 1.199 & $.359,2.038$ & .005 \\
\hline & & & & Calsurf & 0.074 & $-.433, .581$ & .774 \\
\hline \multirow{3}{*}{$\begin{array}{l}\text { Hours on oxygen } \\
\text { (hours), mean (SD) }\end{array}$} & \multirow[t]{3}{*}{$192(105)$} & \multirow[t]{3}{*}{$193(99)$} & \multirow[t]{3}{*}{0.862} & Gestational age & 0.502 & $-4.251,5.254$ & .836 \\
\hline & & & & Antenatal steroid & -31.261 & $-67.681,5.160$ & .092 \\
\hline & & & & Calsurf & 4.485 & $-17.511,26.481$ & .689 \\
\hline \multirow{3}{*}{$\begin{array}{l}\text { Length of stay (days), } \\
\text { mean (SD) }\end{array}$} & \multirow[t]{3}{*}{$15.1(7.3)$} & \multirow[t]{3}{*}{$14.9(6.1)$} & \multirow[t]{3}{*}{0.834} & Gestational age & -0.070 & $-.387, .246$ & .663 \\
\hline & & & & Antenatal steroid & 1.669 & $-.758,4.097$ & .177 \\
\hline & & & & Calsurf & -0.196 & $-1.664,1.272$ & .793 \\
\hline \multirow[t]{3}{*}{ Pneumothorax, $n(\%)$} & \multirow[t]{3}{*}{$28(17.3)$} & \multirow[t]{3}{*}{$25(15.1)$} & \multirow[t]{3}{*}{0.501} & Gestational age & 1.048 & $.921,1.191$ & .479 \\
\hline & & & & Antenatal steroid & 0.359 & $.113,1.387$ & .147 \\
\hline & & & & Calsurf & 0.825 & $.454,1.500$ & .529 \\
\hline \multirow[t]{3}{*}{ Grade 3/4 IVH and/or PVL, $n(\%)$} & \multirow[t]{3}{*}{$15(9.2)$} & \multirow[t]{3}{*}{$9(5.4)$} & \multirow[t]{3}{*}{0.153} & Gestational age & 1.026 & $.859,1.226$ & .776 \\
\hline & & & & Antenatal steroid & 0.724 & $.154,3.417$ & .684 \\
\hline & & & & Calsurf & 0.539 & $.227,1.278$ & .161 \\
\hline \multirow[t]{3}{*}{ Pumonary Hypertension, $n(\%)$} & \multirow[t]{3}{*}{$9(5.6)$} & \multirow[t]{3}{*}{$6(3.6)$} & 0.380 & Gestational age & 0.839 & $.658,1.071$ & .158 \\
\hline & & & & Antenatal steroid & 1.969 & $.383,10.112$ & .417 \\
\hline & & & & Calsurf & 0681 & $.231,2.010$ & .487 \\
\hline Necrotizing enterocolitis & $1(0.6)$ & $0(0)$ & 0.306 & Gestational age & -1.305 & $.007,10.340$ & .482 \\
\hline stage 2 or higher, $n(\%)$ & & & & Antenatal steroid & 16.194 & .000 & .998 \\
\hline & & & & Calsurf & 15.111 & .000 & .996 \\
\hline
\end{tabular}

$I V H$, intraventricular hemorrhage; $P V L$, periventricular leukomalacia. Values are number (percentage) unless otherwise indicated. $P>0.05$ for all comparisons. Ventilator free days $=28$-x, if the patient successfully weaned from mechanical ventilation within 28 days, where the $x$ is the number of days spent receiving mechanical ventilation; Ventilator free days $=0$, if the patient dies before 28 days or of the patient requires mechanical ventilation for more than 28 days

clinicians performed several single center clinical studies (publishing their data in Chinese journals), and concluded that both low $(40 \mathrm{mg} / \mathrm{kg})$ and high( $(100 \mathrm{mg} / \mathrm{kg})$ dose Calsurf manifested similar curative effects on mild to moderate RDS' however, for severe RDS, higher dose Calsurf showed better results[18, 19]. Thus, we used the higher dose of bovine surfactants in the current study, and showed that the OI in the Calsurf group was significantly lower than in the control group after surfactant administration. In the stratified study, the results were equivalent in mild, moderate, and severe cases of NARDS, and these results are somewhat consistent with those reported by Wilson and Luo, who demonstrated that surfactant acutely improved oxygenation and significantly decreased mortality in children and adolescents with ARDS [20, 21].

The mortality rate of infants with NARDS was proximately $5 \%$ in our study, while severe NARDS manifested the highest mortality rate $(15 \%)$. The rate of complications did not differ between the two groups, and some investigators have suggested that the mortality rate in children is lower than in adults-ranging between $18 \%$ and $27 \%$ [22]. Wong reported that mortality was approximately $30.3 \%$ in his study of pediatric patients with ARDS [23], while in our study we found that the mortality rate in infants with NARDS was much lower than in older children. There have no differences in the secondary outcomes as these are extremely multifactorial and it is difficult that surfactant can change this.

\section{Limitations}

To the best of our knowledge, this is the first study to describe the effects of surfactant in treating NARDS. However, there are limitations to this study. First, for preterm infants under 34 weeks of gestation, clinicians have some difficulties to the differential definition of neonatal RDS and NARDS, thus to standardize the definition for all the 12 NICUs, preterm babies under 34 weeks of gestation 
were not included in our study. The mortality rate recorded herein, therefore, might not reflect the overall NARDS. Second, there are two kinds of surfactants in Chinese market: bovine surfactant and porcine surfactant. Exclusively bovine surfactants were used in this study; however, the results could not be applied to other surfactants. Thirdly, because there is lack of phase I/II studies about Calsurf dose range, we do not know whether $100 \mathrm{mg} / \mathrm{kg}$ is the best dose for treating NARDS, thus more dose ranging studies for Calsurf are needed. Moreover, the lack of the information about SNAPPE-II score is another limitation, since it is a better indicator to compare the severity of the disease between the two groups. In addition, not every center can provide inhaled NO and ECMO, so these two treatments were not assessed in the current study.

\section{Conclusion}

Calsurf acutely improved oxygenation immediately after administration in pneumonia-induced NARDS at a gestational age beyond 34 week. However, we recommend that surfactant should be used cautiously in pneumonia-induced NARDS.

Acknowledgments We thank Dr Cong Liu for the review of the statistical analysis in this manuscript preparation.

Authors' contributions Dr. Zhihui Rong conceptualized and designed the study, drafted the initial manuscript, and reviewed and revised the manuscript.

Dr. Luxia Mo designed the data collection instruments, drafted the initial manuscript, and reviewed and revised the manuscript.

Dr. Rui Pan designed the data collection instruments, drafted the initial manuscript, and reviewed and revised the manuscript.

Dr. Xiaofang Zhu designed the data collection instruments, and reviewed and revised the manuscript.

Dr. Hongbin Cheng designed the data collection instruments, and reviewed and revised the manuscript.

Dr. Maojun Li designed the data collection instruments, and reviewed and revised the manuscript.

Dr. Lubiao Yan designed the data collection instruments, and reviewed and revised the manuscript.

Dr. Yujie Lang designed the data collection instruments, and reviewed and revised the manuscript.

Dr. Xiaoshan Zhu designed the data collection instruments, and reviewed and revised the manuscript.

Dr. Liping Chen designed the data collection instruments, and reviewed and revised the manuscript.

Dr. Shiwen Xia designed the data collection instruments, and reviewed and revised the manuscript.

Dr. Jun Han designed the data collection instruments, and reviewed and revised the manuscript.

Profs. Liwen Chang helped in the study design, preparation of data collection instrument, reviewed all the data and performed their interpretation, drafted the manuscript, and significantly reviewed and revised it. All authors approved the final version manuscript.
Funding This study was supported by the research clinicians of Huazhong University of Science and Technology (Second batch) and China International Medical Foundation 2018.

\section{Compliance with ethical standards}

Conflict of interest The authors declare that they have no conflicts of interest.

Ethical approval All procedures described herein were followed in accordance with the ethical standards of the responsible committee on human experimentation (institutional and national) and with the Helsinki Declaration of 1975, as revised in 2000 (5).

Informed consent Informed consent was obtained from all individual participants included in the present study.

\section{References}

1. Cheifetz IM. Pediatric ARDS. Respir Care 2017;62(6):718-731.

2. Phung TTB, Suzuki T, Phan PH (2017 Nov) Pathogen screening and prognostic factors in children with severe ARDS of pulmonary origin. Pediatr Pulmonol 52(11):1469-1477

3. Heidemann SM, Nair A, Bulut Y, Sapru A (2017) Pathophysiology and management of acute respiratory distress syndrome in children. Pediatr Clin N Am 64(5):1017-1037

4. De Luca D, van Kaam AH, Tingay DG (2017) The Montreux definition of neonatal ARDS: biological and clinical background behind the description of a new entity. Lancet Respir Med 5(8): 657-666

5. Fenton TR, Nasser R, Eliasziw M, Kim JH, Bilan D, Sauve R (2013) Validating the weight gain of preterm infants between the reference growth curve of the fetus and the term infant. BMC Pediatr 13:92

6. Claireaux AE (1953) Hyaline membrane in the neonatal lung. Lancet 262:749-753

7. Luo J, Chen J, Li Q, Feng Z (2019) Differences in clinical characteristics and therapy of neonatal acute respiratory distress syndrome (ARDS) and respiratory distress syndrome (RDS): a retrospective analysis of 925 cases. Med Sci Monit 25:4992-4998

8. Khemani RG, Smith LS, Zimmerman JJ, Erickson S (2015) for the Pediatric Acute Lung Injury Consensus Conference Group. Pediatric acute respiratory distress yndrome: definition, incidence, and epidemiology: proceedings from the Pediatric Acute Lung Injury Consensus Conference. Pediatr Crit Care Med 16(5 suppl 1):S23-S40

9. Günther A, Ruppert C, Schmidt R, Markart P, Grimminger F, Walmrath D, Seeger W (2001) Surfactant alteration and replacement in acute respiratory distress syndrome. Respir Res 2:353-364

10. Touqui L, Arbibe L (1999) A role for phospholipase A2 in ARDS pathogenesis. Mol Med Today 5:244-249

11. Spragg RG, Lewis JF, Walmrath H-D et al (2004) Effect of recombinant surfactant protein C-based surfactant on the acute respiratory distress syndrome. N Engl J Med 351(9):884-92. 98

12. Taut FJH, Rippin G, Schenk P, Findlay G, Wurst W, Häfner D, Lewis JF, Seeger W, Günther A, Spragg RG (2008) A search for subgroups of patients with ARDS who may benefit from surfactant replacement therapy: a pooled analysis of five studies with recombinant surfactant protein-C surfactant (Venticute). Chest 134(4): 724-732

13. Kong X, Cui Q, Hu Y, Huang W, Ju R, Li W, Wang R, Xia S, Yu J, Zhu T, Feng Z (2016) Bovine surfactant replacement therapy in 
neonates of less than 32 weeks' gestation: a multicenter controlled trial of prophylaxis versus early treatment in China-a pilot study. Pediatr Neonatol 57(1):19-26

14. Liu J, Yang N, Liu Y (2014) High-risk factors of respiratory distress syndrome in term neonates: a retrospective case-control study. Balkan Med J 31(1):64-68

15. Cogo PE, Facco M, Simonato M, de Luca D, de Terlizi F, Rizzotti U, Verlato G, Bellagamba MP, Carnielli VP (2011) Pharmacokinetics and clinical predictors of surfactant redosing in respiratory distress syndrome. Intensive Care Med 37(3):510-517

16. Singh N, Halliday HL, Stevens TP, Suresh G, Soll R, Rojas-Reyes MX (2015) Comparison of animal-derived surfactants for the prevention and treatment of respiratory distress syndrome in preterm infants. Cochrane Database Syst Rev (12):CD010249. Published 2015 Dec 21. https://doi.org/10.1002/14651858.CD010249

17. Tridente A, De Martino L, De Luca D (2019) Porcine vs bovine surfactant therapy for preterm neonates with RDS: systematic review with biological plausibility and pragmatic meta-analysis of respiratory outcomes. Respir Res 20(1):28. Published 2019 Feb 6. https://doi.org/10.1186/s12931-019-0979-0

18. Liu J et al (2014) Clinical evaluation of Calsurf in treating full-term neonatal respiratory distress syndrome. Chin Pediatr Emerg Med 21(5):259-262
19. Wang XH et al (2014) Different dosage of bovine surfactant in treating respiratory distress syndrome in late preterm infants. Chin J Neonatol 29(1):43-45

20. Willson DF, Thomas NJ, Markovitz BP et al (2005) Effect of exogenous surfactant (calfactant) in pediatric acute lung injury: a randomized controlled trial. JAMA. 293(4):470-476

21. Möller JC, Schaible T, Roll C et al (2003) Treatment with bovine surfactant in severe acute respiratory distress syndrome in children: a randomized multicenter study. Intensive Care Med 29(3):437446

22. Rotta AT, Piva JP, Andreolio C et al (2015) Progress and perspectives in pediatric acute respiratory distress syndrome. Rev Bras Ter Intensiva 27(3):266-273

23. Wong JJ, Phan HP, Phumeetham S et al (2017) Risk stratification in pediatric acute respiratory distress syndrome: a multicenter observational study. Crit Care Med 45(11):1820-1828

24. Dell'Orto V, Bourgeois-Nicolaos N, Rouard C et al (2018) Cell count analysis from nonbronchoscopic bronchoalveolar lavage in preterm infants. J Pediatr 200:30-37.e2. https://doi.org/10.1016/j. jpeds.2018.04.074

Publisher's note Springer Nature remains neutral with regard to jurisdictional claims in published maps and institutional affiliations. 


\section{Affiliations}

\section{Zhihui Rong ${ }^{1}$ (1D $\cdot{\text { Luxia } \mathrm{Mo}^{2} \cdot \text { Rui Pan }^{3} \cdot \text { Xiaofang Zhu }^{4} \cdot \text { Hongbin Cheng }}^{5} \cdot$ Maojun $\mathrm{Li}^{6} \cdot$ Lubiao Yan $^{7} \cdot$ Yujie Lang $^{8}$. Xiaoshan Zhu ${ }^{9} \cdot$ Liping Chen $^{10} \cdot$ Shiwen Xia ${ }^{11}$. Jun Han ${ }^{12} \cdot$ Liwen Chang $^{1}$}

Zhihui Rong

rongzhihui53@163.com

Luxia Mo

molux87@126.com

Rui Pan

pc79926com@163.com

Xiaofang Zhu

649491619@qq.com

\section{Hongbin Cheng}

1340333241@qq.com

Maojun Li

hanman2012@163.com
Lubiao Yan

ylb2003@163.com

Yujie Lang

jn_langyujie@sina.com

Xiaoshan Zhu

13855111997@163.com

Liping Chen

ccllpp88@qq.com

Shiwen Xia

shiwenxia66@163.com

Jun Han

15804302910@139.com
1 Department of Neonatology, Tongji Hospital, Tongji Medical College, Huazhong University of Science and Technology, Wuhan 430030, China

2 Department of Neonatology, Wuhan Maternal and Child Healthcare Hospital, Tongji Medical College, Huazhong University of Science \& Technology, Wuhan, China

3 Department of Neonatology, Xiangyang Central Hospital, Affiliated Hospital of Hubei University of Arts and Science, Xiangyang, China

4 Department of Neonatology, Jingzhou Central Hospital, Jingzhou, China

5 Department of Neonatology, HuangShi Maternal and Child Healthcare Hospital, Huangshi, China

6 Department of Neonatology, Sichuan Academy of Medical Sciences and Sichuan Provincial People's Hospital, Sichuan, China
7 Department of Neonatology, Maternal and Child Healthcare Hospital, Nanjing Medical University, Nanjing, China

8 Department of Neonatology, Children's Hospital of Jinan, Jinan, China

9 Department of Neonatology, Anhui Provincial Children's Hospital, Hefei, China

10 Department of Neonatology, Jiangxi Provincial Children's Hospital, Jiangxi, China

11 Department of Neonatology, Hubei Maternity and Child Heath Hospital, Wuhan, China

12 Department of Neonatology, The First Hospital of Jilin University, Changchun, China 